Sharif University of Technology
Scientia Iranica
SCIENTIA

Invited Paper

\title{
Decentralized energy trading framework for active distribution networks with multiple microgrids under uncertainty
}

\author{
M. Doostizadeh*, M.R. Shakarami, and H. Bastami \\ Faculty of Engineering, Lorestan University, 5 km Tehran Road, Khorramabad, P.O. Box 68151-44316, Lorestan, Iran. \\ Received 17 July 2019; accepted 14 September 2019
}

\author{
KEYWORDS \\ Active distribution \\ network; \\ Analytical target \\ cascading; \\ Decentralized energy \\ trading; \\ Multiple microgrids; \\ Parallel algorithm.
}

\begin{abstract}
The ever-increasing need for more reliable power supply as well as cost-effective and environmentally friendly utilization of distributed energy resources will result in the formation of Multiple Micro-Grids (MMGs) in the near future of distribution system. To reach this prospect, coordination among MMGs is necessary. Accordingly, this paper proposes a new non-hierarchical multi-level architecture for the optimal scheduling of Active Distribution Network (ADN) with MMGs. The proposed model is a decentralized decision making algorithm to optimally coordinate the mutual interaction between local optimization problems of ADN and MMGs. A non-hierarchical Analytical Target Cascading (ATC) method is presented to solve the local optimization problems in parallel. Also, the underlying risks of energy trading caused by renewable generation uncertainty are reflected in both the objective functions and the constraints of local optimization problem. The numerical results of modified IEEE 33-bus distribution test system containing two microgrids demonstrate the effectiveness and merits of the proposed model.
\end{abstract}

(C) 2019 Sharif University of Technology. All rights reserved.

\section{Introduction}

Micro-Grids (MGs) are small-scale electrical networks with a defined border integrating various types of renewable and non-renewable Distributed Generations (DGs), Energy Storage Systems (ESSs), and controllable loads. MGs may act as either a power source or a load when they are connected to an Active Distribution Network (ADN) or they can work individually during islanded operation. These features have a great significance in promoting economic resiliency and reliability

\footnotetext{
*. Corresponding author. Tel.: +9866 33120097;

Fax: +986633120104

E-mail addresses: doostizadeh.m@lu.ac.ir (M. Doostizadeh)

shakarami.mr@lu.ac.ir (M.R.Shakarami)

bastami.hu@fe.lu.ac.ir (H.Bastami)
}

of ADNs $[1,2]$. Given the increasing growth of MGs in ADNs, the coordinated operation of ADN and several MGs has become a new research hotspot. Conventionally, distribution system operator has the decision authority to operate the entire distribution system. However, ADN and MGs are autonomous entities with their own rules and policies, each of which solves its local energy management problem with the goal of maximizing its benefits [3]. Therefore, only limited information between ADN and MGs can be exchanged for privacy and security concerns. Moreover, operation of AND with Multiple MGs (MMGs) becomes more challenging when different types of uncertainty such as wind and solar generation, and electrical vehicles are noticed [4]. Considering several security, privacy, and uncertainty issues, coordinated operation of ADN with MMGs is one of the most challenging tasks of modern power systems. 
Several studies of integrated MGs, which are also named networked MGs, have so far been presented in the literature. With regard to the energy management techniques used in the operation of networked MGs, these researches can be categorized into two groups with centralized [5-9] and decentralized [10-33] approaches. In the centralized optimization approach, all entities are gathered as one system with a united objective and a central controller is designed to manage the operation of all resources and loads irrespective of their individual interests. From this viewpoint, a cooperative power dispatch algorithm for MMGs with the objective of grid operational cost minimization was presented by Fathi and Bevrani [5]. Ouammi et al. [6] presented a Model Predictive Control (MPC) strategy to determine the scheduling of power exchanges among MGs and their internal resources with the aim of maximizing global benefits. In the studies carried out by Haddadian and Noroozian [7,8], technical indices for quality evaluation of ADN were presented to achieve optimal operation of ADN with MMGs. In order to obtain the scheduling scheme, which minimized operating cost of MMGs under renewable generation uncertainties, a two-stage robust optimization model was presented by Zhang et al. [9]. However, centralized scheme requires a massive communication system for data exchange, leads to high computation burden when scale of the system is large, barely covers different interests of the ADN and MGs, and is more vulnerable to cyber-attack [10]. Hence, decentralized approaches have been proposed to address these shortcomings.

In some studies [11-13], bilevel optimization problems have been proposed to model the interactions between the ADN in the upper level and MGs in the lower level, where MGs are modeled as nodes in the $\mathrm{ADN}$ and their internal power flow is neglected. Also, the uncertainties related to demand and renewable generation are captured by the scenario-based two-stage stochastic optimization approach. Some theoretical game strategies have also been presented in the literature [14-19] for operation of ADN with multiple MGs. An incentive mechanism using Nash negotiating theory was suggested in Wang and Huang [14] to encourage energy trading and fair profit sharing among MGs. Contribution-based and priority-based energy-trading strategies among MGs in a competitive environment were designed by Park et al. [15] and Jadhav and Patne [16], respectively. These strategies were nonpricing-based approaches and could only be applied to buyer MGs. Cooperative and coalitional approaches to local power exchange in networked MGs were presented by $\mathrm{Du}$ et al. [17] and Mei et al. [18], respectively. Moreover, a collaborative Stackelberg game strategy was established by Liu et al. [19] to promote power trading among MGs. The uncertainties caused by renewable resources and demand were handled through a two-stage robust optimization model. Although these strategies consider the local power exchanges for both buyer MGs and seller MGs, network constraints of the MGs are ignored. In addition to the theoretical game strategies, several methods based on multiagent system have been developed [20-22] to achieve decentralized control in the energy management of MMG systems. A distributed MPC strategy was also suggested by Kou et al. [23] and Holjevac et al. [24] to maintain a stable power interchange among MGs under the uncertainties of renewable energy and load.

From another perspective, coordinated operation of ADN and multiple MGs has been envisioned in recent researches by utilizing distributed optimization techniques such as Alternating Direction Method of Multipliers (ADMM) [25-28], Dantzig-Wolfe decomposition [29], proximal message passing [30], Analytical Target Cascading (ATC) [31-33], etc. These algorithms typically decompose the whole problem into several subproblems to distribute computation and reduce data communication [34]. For example, ADMMbased methods consider each MG as a distinct interest subject. Then, the objective function of each MG is accompanied by an augmented Lagrangian penalty term to model the interactive electric power of tielines and economic scheduling of ADN with MMGs is determined iteratively. In ATC method, the entire system is decomposed hierarchically into subsystems in decreasing order. Then, each subsystem within the hierarchy is coupled with the lower/higher-level subsystem through target/response variables. Target and response consistencies are relaxed as penalty functions in the optimization problem of the subsystem and the optimal solution is iteratively obtained. In a comparison of several algorithms for distributed/decentralized optimization in power systems carried out by Kargarian et al. [34], it was shown that ATC and ADMM needed a low amount of information exchange between subproblems per iteration and they fairly protected the privacy of subproblems. However, the computational effort with these algorithms was relatively high. Moreover, both the classical ADMM and ATC needed a central coordinator that made them potentially vulnerable to cyber-attacks or manipulation of information. Compared with ADMM algorithm, ATC had flexibilities in the choice of penalty functions and coordination of subproblems. Moreover, solution time of ATC method was sensibly lower than that of ADMM [34].

In the above review of literature, it is notable that there are three concerns in the development of coordinated operation of ADN with MMGs. The first one is the optimal energy trading among ADN and MMGs. The second issue is the decentralized control architecture, which gives independence to each MG. The last one is the uncertainty management of MGs operation. Motivated by the aforementioned research 
gap, this paper proposes a decentralized decision making algorithm that allows parallel implementation of local optimization problems of ADN and MGs without the necessity for any kind of central coordinator to synchronize the local problems. The proposed algorithm allows direct communication between any two local problems. Thus, it is potentially less vulnerable to failure in information exchange and cyber-attacks than the classical ATC is with central coordinator. The main contributions of this paper are as follows:

- A non-hierarchical ATC algorithm is proposed for decentralized optimal transactive energy implementation in ADN with MMGs. The proposed algorithm allows parallel implementation of local optimization problems without the need for any form of central coordinator. Therefore, the proposed algorithm achieves the same accuracy and convergence as hierarchical ATC does, while it is less vulnerable to cyber-attacks and data manipulation, and requires much less computational effort;

- A fully distributed second-order conic programming method is proposed to achieve an exact model of the network of MGs using the AC optimal power flow, which, to the best of our knowledge, has not been considered in the literature. The proposed method ensures that local energy trading between ADN and MGs is accounted for in the OPF-based energy management systems. Therefore, internal network limits of MGs are explicitly considered while very little boundary information is exchanged;
- A risk-based day-ahead scheduling model is established in each local problem to capture the underlying risks of the scheduling imposed by multiple source-load uncertainties. The risks are calculated based on the model-free probabilistic distributions of net demand and considered as penalties in the objective functions as well as limits of risks in the constraints. The nonlinear terms related to the risks are linearized, and the proposed model is transformed to a Mixed-Integer Second-Order Conic Programming (MISOCP) problem.

The remainder of this paper is organized as follows: Section 2 describes the structure of the ADN with MMGs and presents the mathematical formulation of the optimization problem for ADN and MGs. Section 3 presents the proposed non-hierarchical ATC algorithm. The simulation results for a modified IEEE 33 distribution test system are presented in Section 4 to show the efficiency of the proposed algorithm. Finally, conclusions are given in Section 5 .

\section{System architecture and modeling}

\subsection{System architecture}

In this paper, the ADN under study contains several MGs connected to different nodes, in which energy trading between ADN and MGs is allowed. As depicted in Figure 1, each MG may have several Wind Turbines (WTs), Photovoltaics (PVs), Controllable DGs (CDGs), battery ESSs, and local loads. Each MG

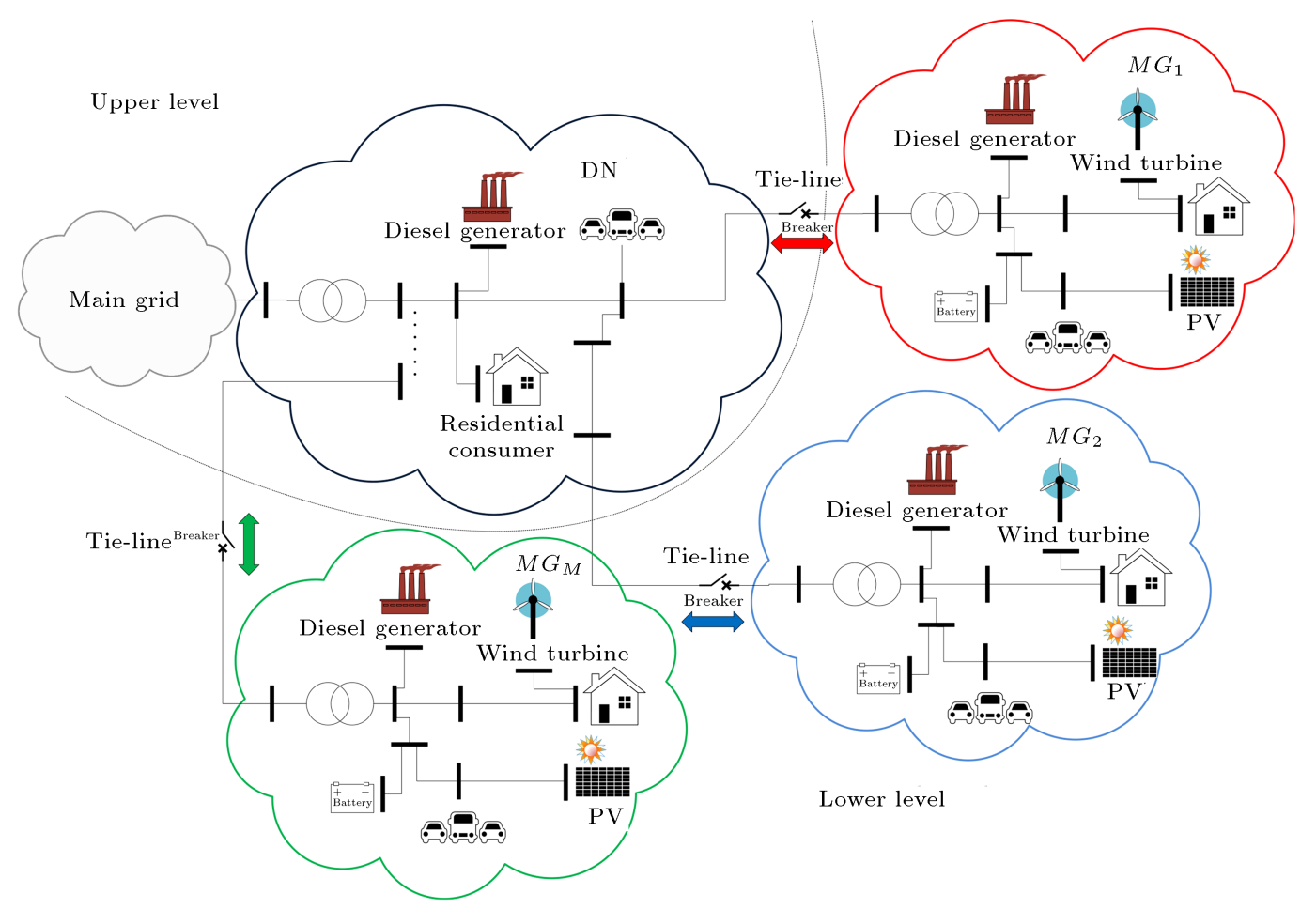

Figure 1. An active distribution network comprising multiple MGs. 
operator is required to provide nodal power balance, meet resource limitations, and secure network operation. Distribution Network (DN) operator is responsible for satisfying DN-owned generation resources, system operation safety, and reliable power supply of its loads. Moreover, both ADN and MGs are expected to minimize their own cost. As well, power exchange via the tie-lines strongly couples their scheduling problems. Thus, they are required to share boundary information with each other via a communication infrastructure to bidirectionally determine the optimal energy trading. Meanwhile, the DN can interchange energy with the main grid.

\subsection{Mathematical formulation}

This section presents the local optimization problems of DN and MGs under the uncertainties of renewable energy and load. To do so, we describe all parts of operation cost, all the constraints of the optimization problem, and the uncertainty modeling for both DN and MGs. Then, the proposed non-hierarchical ATC algorithm for energy interchange coordination of DN and MGs will be presented in Section 3. The variables and parameters defined in the proposed model are listed and described in Nomenclature. To avoid duplications, variables and parameters are specified by superscript $A \in\left\{\mathrm{DN}, \mathrm{MG}_{1}, \mathrm{MG}_{2}, \ldots, \mathrm{MG}_{M}\right\}$, which means that the mathematical formulations are applied to both DN and MGs.

\subsubsection{Cost functions and objectives}

In the proposed model, both DN and MGs aim to minimize their local operation costs, including generation cost for CDGs $\left(\cos t_{C D G s}^{A}\right)$, ESSs operation cost $\left(\cos t_{E S S s}^{A}\right)$, operating reserves cost $\left(\cos t_{R e s}^{A}\right)$, importing power from the main grid that is only applied to DN $\left(\cos t_{P s}^{D N}\right)$, and underlying risk costs associated with renewable generation and load uncertainties $\left(\cos t_{\text {Risk }}^{A}\right)$. Therefore, the objective function can be expressed by Eq. (1) as shown in Box I.

\subsection{2. $C D G$ s operating constraints}

Each CDG has several operating limitations that are represented by Eqs. (2)-(12). Constraints (2) and (3) respectively guarantee the minimum up and down time limits. The power output plus the reserve of a CDG is limited by Eqs. (4) and (5). Maximum upward and downward reserves provided by each CDG are respectively restricted via Eqs. (6) and (7). Constraints (8) and (9) represent CDG downward and upward ramping capability limits, respectively. Reactive power limitations and the linearized form of quadratic relation between active and reactive power for each CDG are represented by Eqs. (10)-(12).

$$
\begin{gathered}
\sum_{t=1}^{T_{i}^{O N}}\left(1-u_{i, t}^{D G(A)}\right)=0 \\
\sum_{h=t}^{t+U T_{i}-1} u_{i, h}^{D G(A)} \geq U T_{i}\left(u_{i, t}^{D G(A)}-u_{i, t-1}^{D G(A)}\right) \\
\forall t=T^{O N}+1, \cdots, N T-U T_{i}+1, \\
\sum_{h=t}^{N T}\left[u_{i, h}^{D G(A)}-\left(u_{i, t}^{D G(A)}-u_{i, t-1}^{D G(A)}\right)\right] \geq 0 \\
\forall t=N T-U T_{i}+2, \cdots, N T,
\end{gathered}
$$

$$
\begin{aligned}
& \cos t_{\text {Tot }}^{A}=\underbrace{\sum_{t \in T} \sum_{i \in S u b(D N)}\left(C_{i, t}^{S u b(D N)} \times P_{i, t}^{S u b(D N)}\right)}_{\cos t_{P S}^{D N}(\text { only for } D N)} \\
& +\underbrace{\sum_{t \in T} \sum_{i \in D G(A)}\left(a_{i, t}^{D G(A)} \times\left(P_{i, t}^{D G(A)}\right)^{2}+b_{i, t}^{D G(A)} \times P_{i, t}^{D G(A)}+c_{i, t}^{D G(A)}\right)}_{\cos t_{D G s}^{A}} \\
& +\underbrace{\sum_{t \in T} \sum_{i \in D G(A)}\left(C r_{i, t}^{u p(A)} \times R_{i, t}^{u p(A)}+C r_{i, t}^{d n(A)} \times R_{i, t}^{d n(A)}\right)}_{\cos t_{R e s}^{A}}+\underbrace{\sum_{t \in T} \sum_{i \in E S S(A)}\left(C_{i, t}^{S C(A)} \times P_{i, t}^{S C(A)}+C_{i, t}^{S D(A)} \times P_{i, t}^{S D(A)}\right)}_{\cos t_{E S S}^{A}} \\
& +\underbrace{\sum_{t \in T}\left(C_{t}^{E R C(A)} \times R_{t}^{E R C(A)}+C_{t}^{E E N S(A)} \times R_{t}^{E E N S(A)}\right)}_{\cos t_{\mathrm{Risk}}^{A}} .
\end{aligned}
$$




$$
\begin{aligned}
& \sum_{t=1}^{T_{i}^{O F F}} u_{i, t}^{D G(A)}=0 \\
& \sum_{h=t}^{t+D T_{i}-1}\left(1-u_{i, h}^{D G(A)}\right) \geq D T_{i}\left(u_{i, t-1}^{D G(A)}-u_{i, t}^{D G(A)}\right) \\
& \forall t=T^{\mathrm{OFF}}+1, \cdots, N T-D T_{i}+1, \\
& \sum_{h=t}^{N T}\left[\left(1-u_{i, h}^{D G(A)}\right)-\left(u_{i, t-1}^{D G(A)}-u_{i, t}^{D G(A)}\right)\right] \geq 0 \\
& \forall t=N T-D T_{i}+2, \cdots, N T, \\
& P_{i, t}^{D G(A)}+R_{i, t}^{u p(A)} \leq \bar{P}_{i}^{D G(A)} \times u_{i, t}^{D G(A)}, \\
& P_{i, t}^{D G(A)}-R_{i, t}^{d n(A)} \leq \underline{P}_{i}^{D G(A)} \times u_{i, t}^{D G(A)}, \\
& R_{i, t}^{u p(A)} \leq \bar{R}_{i}^{u p(A)}, \\
& R_{i, t}^{d n(A)} \leq \bar{R}_{i}^{d n(A)}, \\
& P_{i, t-1}^{D G(A)}-P_{i, t}^{D G(A)} \leq R D_{i}^{D G(A)}, \\
& P_{i, t}^{D G(A)}-P_{i, t-1}^{D G(A)} \leq R U_{i}^{D G(A)}, \\
& \underline{\mathrm{Q}}_{i}^{D G(c)} \times u_{i, t}^{D G(A)} \leq Q_{i, t}^{D G(c)} \leq \bar{Q}_{i}^{D G} \times u_{i, t}^{D G(A)}, \\
& P_{i, t}^{D G(A)}+Q_{i, t}^{D G(A)} \leq \sqrt{2} \bar{S}_{i}^{D G(A)}, \\
& P_{i, t}^{D G(A)}-Q_{i, t}^{D G(A)} \leq \sqrt{2} \bar{S}_{i}^{D G(A)} .
\end{aligned}
$$

\subsubsection{ESSs constraints}

In this paper, it is assumed that ESSs are connected to the network during the operating day, and they should meet the following constraints for secure operation and periodic deployment.

$$
\begin{gathered}
0 \leq P_{i, t}^{S D(A)} \leq \bar{P}_{i}^{S D(A)} \times u_{i, t}^{S D(A)}, \\
0 \leq P_{i, t}^{S C(A)} \leq \bar{P}_{i}^{S C(A)} \times u_{i, t}^{S C(A)}, \\
u_{i, t}^{S C(A)}+u_{i, t}^{S D(A)} \leq 1, \\
E_{i, t}^{S(A)}=E_{i, t-1}^{S(A)}-\frac{1}{\eta_{i}^{S D(A)}} P_{i, t}^{S D(A)} \Delta T \\
\quad+\eta_{i}^{S C(A)} P_{i, t}^{S C(A)} \Delta T, \\
\underline{E}_{i}^{S(A)} \leq E_{i, t}^{S(A)} \leq \bar{E}_{i}^{S(A)}
\end{gathered}
$$

where Eqs. (13) and (14) are the maximum dis- charge/charge power constraints; Eq. (15) determines the charging and discharging status; the current energy stored in ESS is calculated via Eq. (16); and Eq. (14) indicates that the energy level of ESS should be within specific limits.

\subsubsection{Underlying risks of renewable output and demand}

As shown in Eq. (1), the proposed model considers the underlying risk costs related to renewable output curtailment and loss of load. In order to calculate the Expected Renewable Curtailment (ERC) and Expected Energy Not Supplied (EENS), the deficiencies of downward and upward reserves are respectively multiplied by the integration of the probability of net demand $\left(f_{\text {net, } \mathrm{t}}^{A}(x)\right)$ (i.e., load minus renewable generation output) as follows:

$$
\begin{aligned}
& D_{t}^{n e t(A)}+\sum_{i \in D G(A)} R_{i, t}^{u p(A)} \\
& R_{t}^{E R C(A)}=\int_{\underline{D}_{t}^{\text {net }(A)}} \\
& \left(D_{t}^{\text {net }(A)}+\sum_{i \in D G(A)} R_{i, t}^{u p(A)}-x\right) f_{n e t, t}^{A}(x) d x, \\
& R_{t}^{E E N S(A)}=\int_{D_{t}^{n e t(A)}+\sum_{i \in D G(A)} R_{i, t}^{d n(A)}}^{\bar{D}_{t}^{n e t(A)}} \\
& \left(x-D_{t}^{n e t(A)}+\sum_{i \in D G(A)} R_{i, t}^{d n(A)}\right) \times f_{n e t, t}^{A}(x) d x, \\
& D_{t}^{n e t(A)}=P_{t}^{L(A)}-P_{t}^{W(A)}-P_{t}^{P V(A)},
\end{aligned}
$$

where $\bar{D}_{t}^{\text {net( }(A)}$ and $\underline{D}_{t}^{\text {net( }(A)}$ are respectively the possible maximum and minimum net demands, and $D_{t}^{\text {net }(A)}$ is the forecasted net demand during hour $t$. It should be noted that the possible scenarios for net demand are not limited to the well-known probability distribution functions in the proposed model and it can follow any ambiguous distributions with known expectations and variances. Thus, $f_{n e t, t}^{A}(x)$ can be calculated using the available historical data. However, Eq. (18) is yet difficult to calculate due to the lack of exact distribution information as well as nonlinearity of $R_{t}^{E R C(A)}$ and $R_{t}^{E E N S(A)}$. As it is depicted in Figure 2, a piecewise linear approximation method [35] is applied to obtaining the approximate linear form of Eq. (18). Note that since $R_{t}^{E R C(A)}$ and $R_{t}^{E E N S(A)}$ are included in the objective function (1), the equality constraints are transformed into inequality ones and rewritten as follows: 


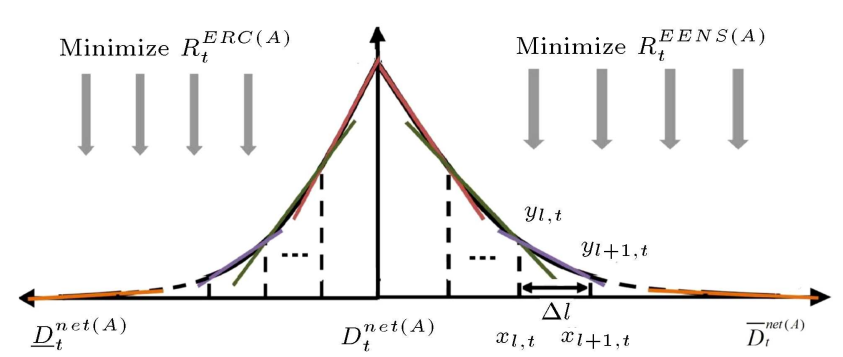

Figure 2. Piecewise linear approximation of risks.

$$
\begin{aligned}
& R_{t}^{E R C(A)} \geq a_{l, t}^{E R C(A)} \sum_{i \in D G(A)} R_{i, t}^{d n(A)}+b_{l, t}^{E R C(A)} \\
& R_{t}^{E E N S(A)} \geq a_{l, t}^{E E N S(A)} \sum_{i \in D G(A)} R_{i, t}^{u p(A)}+b_{l, t}^{E E N S(A)}
\end{aligned}
$$

where $a_{l, t}^{E R C(A)}, a_{l, t}^{E E N S(A)}, b_{l, t}^{E R C(A)}$, and $b_{l, t}^{E E N S(A)}$ are constant coefficients of the piecewise linear approximation and $l$ is an ordinal number generated during the piecewise linearization. Taking $R_{t}^{E E N S(A)}$ as an instance, the coefficients are determined as follows:

$$
\begin{aligned}
& a_{l, t}^{E E N S(A)}=\frac{y_{l, t}-y_{l+1, t}}{x_{l, t}-x_{l+1, t}}, \\
& b_{l, t}^{E E N S(A)}=-x_{l, t} \times a_{l, t}^{E E N S(A)}+y_{l, t}, \\
& y_{l, t}=\int_{D_{t}^{\text {net }(A)}+x_{l, t}}^{\bar{D}_{t}^{\text {net }(A)}}\left(x-D_{t}^{\text {net }(A)}+x_{l, t}\right) \\
& \quad \times f_{\text {net }, t}^{A}(x) d x .
\end{aligned}
$$

Alongside risk costs added to the objective function, i.e., $\cos t_{\text {Risk }}^{A}$, the operators may plan to guarantee a preferred level of result conservativeness. To do so, Eq. (21) is presented to limit the risks to certain amounts:

$$
\begin{aligned}
R_{t}^{E R C(A)} & \leq \bar{R}_{t}^{E R C(A)}, R_{t}^{\operatorname{EENS}(A)} \\
& \leq \bar{R}_{t}^{\operatorname{EENS}(A)}
\end{aligned}
$$

2.2.5. Power flow equations and network constraints In order to model the power flows in both DN and MGs, the distflow model is applied in this paper. As illustrated by Farivar and Low [36], relaxing the original quadratic equality constraints of distflow model to inequality constraints yields a second-order cone program. Accordingly, Eqs. (22) and (23) provide the active and reactive power balances at each node $i$ in each time period $t$, respectively. Constraint (24) represents the power flow relation between any two connected nodes $i$ and $j$. The conic relaxation of the branch flow equation is shown in Eq. (25).

$$
\begin{aligned}
& P_{i, t}^{s u b(A)}+P_{i, t}^{D G(A)}+P_{i, t}^{W(A)}+P_{i, t}^{P V(A)} \\
& -\sum_{j \in S B(i)} P_{i j, t}^{(A)}+\sum_{k \in R B(i)}\left(P_{i k, t}^{(A)}\right. \\
& \left.-r_{i k}^{(A)} I_{i k, t}^{(A)}\right)-g_{i} V_{i, t}^{(A)}=P_{i, t}^{L(A)}, \\
& Q_{i, t}^{s u b(A)}+Q_{i, t}^{D G(A)}-\sum_{j \in S B(i)} Q_{i j, t}^{(A)} \\
& +\sum_{k \in R B(i)}\left(Q_{i k, t}^{(A)}-x_{i k}^{(A)} I_{i k, t}^{(A)}\right) \\
& -b_{i} u_{i, t}^{(A)}=Q_{i, t}^{L(A)} \\
& V_{i, t}^{(A)}=V_{j, t}^{(A)}-2\left(r_{i j, t}^{(A)} P_{i j, t}^{(A)}+x_{i j, t}^{(A)} Q_{i j, t}^{(A)}\right) \\
& +\left(\left(r_{i j, t}^{(A)}\right)^{2}+\left(x_{i j, t}^{(A)}\right)^{2}\right) I_{i j, t}^{(A)}, \\
& \left\|\begin{array}{c}
2 P_{i j, t}^{(A)} \\
2 Q_{i j, t}^{(A)} \\
V_{i, t}^{(A)}-I_{i j, t}^{(A)}
\end{array}\right\|_{2} \leq V_{i, t}^{(A)}+I_{i j, t}^{(A)} .
\end{aligned}
$$

In addition to the above power flow equations, the allowable limits on voltage and thermal capacity of nodes for each branch are described by Eqs. (26) and (27), respectively.

$$
\begin{aligned}
& \underline{v}_{i, t}^{2(A)} \leq V_{i, t}^{(A)} \leq \bar{v}_{i, t}^{2(A)}, \\
& I_{i, t}^{(A)} \leq \bar{i}_{i}^{(A)} .
\end{aligned}
$$

\section{Decentralized ATC-based implementation of the model}

In this section, we formulate the coordinated energy trading model among DN and MGs within the ATC framework. Traditionally, the ATC method is suitable for multilevel systems. It decomposes the system into subsystems hierarchically. Subsystems in the upper levels are parents and broadcast the design targets to the lower-level subsystems, named the children. In order to meet the design targets, children perform consecutive optimizations and compute the corresponding responses. As shown in Figure 1, DN in the upper level is linked to MGs in the lower level via tie-lines. Since power flow in a tie-line is determined by controlling variables at ending terminals of that tie-line, any decisions made by DN would change the power flow in the tie-line and affect the decision-making process of MG. Thus, the local optimization problems of both DN and MGs, presented in Section 2.2, should include power flow relations corresponding to the tie-lines. To do so, the node sets of DN and MGs are extended to 
include the boundary nodes of their neighbors. For example, DN and $M G_{m}$ share their boundary nodes $a$ and $a^{\prime}$ as shown in Figure 3. Consequently, the local copies of variables associated with the shared nodes $a a^{\prime}$, i.e., $\mathbf{S}_{a a^{\prime}, t}^{(A)}=\left[P_{a a^{\prime}, t}^{(A)} Q_{a a^{\prime}, t}^{(A)} V_{a, t}^{(A)} V_{a^{\prime}, t}^{(A)} I_{a a^{\prime}, t}^{(A)}\right]$ should be all driven to the same values for both neighboring agents. Except for this vector of coupling variables $\left(\mathbf{S}_{a a^{\prime}, t}^{(A)}\right)$, the decision variables in the local optimization problems of both DN and MGs are independent and local.

Solving the local optimization problems of both DN and MGs separately with no coordination might yield different values for $\mathbf{S}_{a a^{\prime}, t}^{(A)}$. However, this occasion is physically infeasible and the obtained solution is impractical. To ensure feasible solution, both DN and MGs need to achieve an agreement on the values of coupling variables. In the ATC technique, the coupling variables are revealed in the form of target variables and response copiers. The target variables are the coupling variables $\left(\mathbf{S}_{a a^{\prime}, t}^{(D N)}\right)$ controlled by $\mathrm{DN}$, and the response copiers are the coupling variables $\left(\mathbf{S}_{a a^{\prime}, t}^{\left(M G_{m}\right)}\right)$ managed by MGs. Note that the response copiers are replicates of the target variables. Hence, a set of consistency constraints $\left(\mathbf{C}_{a a^{\prime}, t}^{D N-M G_{m}}\right)$ is formulated as:

$$
\mathbf{C}_{a a^{\prime}, t}^{D N-M G_{m}}: \mathbf{S}_{a a^{\prime}, t}^{(D N)}-\mathbf{S}_{a a^{\prime}, t}^{\left(M G_{m}\right)}=0 \forall t \in T .
$$

By enforcing Eq. (28) in the local optimization of DN and $M G_{m}$, a feasible solution for the coordinated DN+MGs operation is guaranteed. Then, the consistency constraints are relaxed in the objective functions of both DN and MGs by augmented Lagrangian penalty functions as follows:

$$
\begin{aligned}
\pi^{(D N)} & =\sum_{M G_{m} \in N^{M G}} \sum_{s \in \mathbf{S}_{a a^{\prime}}^{(D N)}} \sum_{t \in T} \\
& \left(\nu_{a a^{\prime}, s, t}^{(D N)} \times\left(S_{a a^{\prime}, s, t}^{(D N)}-S_{a a^{\prime}, s, t}^{\left(M G_{m}\right) *}\right)\right. \\
& \left.+\left\|\omega_{a a^{\prime}, s, t}^{(D N)} \circ\left(S_{a a^{\prime}, s, t}^{(D N)}-S_{a a^{\prime}, s, t}^{\left(M G_{m}\right) *}\right)\right\|_{2}^{2}\right),
\end{aligned}
$$

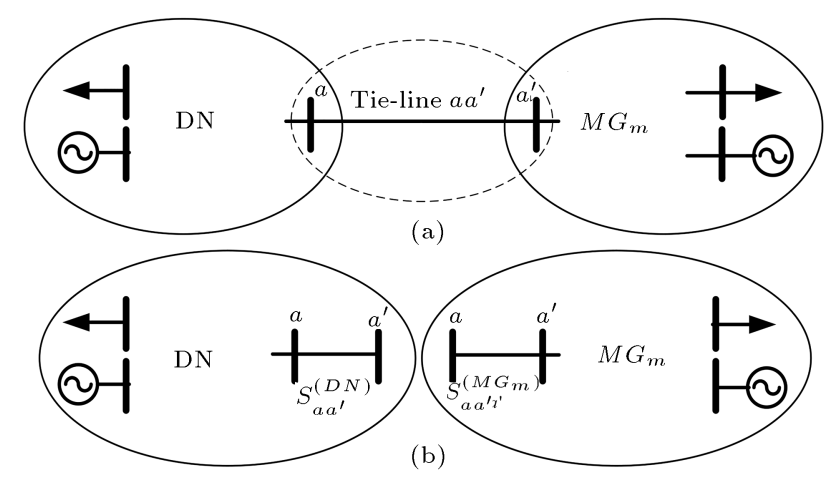

Figure 3. Interdependency of DN and MGs via coupling variables.

$$
\begin{aligned}
\pi^{\left(M G_{m}\right)}= & \sum_{\left.s \in \mathbf{S}_{a a^{\prime}}^{(M G}\right)} \sum_{t \in T} \\
& \left(\nu_{a a^{\prime}, s, t}^{\left(M G_{m}\right)} \times\left(S_{a a^{\prime}, s, t}^{(D N) *}-S_{a a^{\prime}, s, t}^{\left(M G_{m}\right)}\right)\right. \\
& \left.+\left\|\omega_{a a^{\prime}, s, t}^{\left(M G_{m}\right)} \circ\left(S_{a a^{\prime}, s, t}^{(D N) *}-S_{a a^{\prime}, s, t}^{\left(M G_{m}\right)}\right)\right\|_{2}^{2}\right),
\end{aligned}
$$

where $\nu_{a a^{\prime}, s, t}^{(D N)}$ and $\omega_{a a^{\prime}, s, t}^{(D N)}$ are the Lagrange multiplier and the penalty corresponding to the coupling variable $s$ of shared nodes $a a^{\prime}$ at time $t$, respectively. Traditionally, a hierarchical ATC structure can be applied to matching up the local optimization problems. First, the parent (i.e., DN) solves its local optimization problem and transmits the target values $\left(S_{a a^{\prime}, s, t}^{(D N) *}\right)$ to its children (i.e., MGs). Then, the responses $\left(S_{a a^{\prime}, s, t}^{\left(M G_{m}\right) *}\right)$ are determined by solving the local optimization of MGs and sent back to DN. The local optimization problems of DN and MGs are hierarchically linked, while the problems of the MGs are not connected. However, this hierarchical ATC structure is a sequential procedure wherein MGs should wait when $\mathrm{DN}$ is solving its problem, and vice versa. Moreover, any connection/information sharing among MGs is not allowed in traditional ATC. Therefore, a non-hierarchical ATC method with parallelization is proposed in this paper. Instead of transferring target or response between the upper level and the lower level, the results of the previous iteration of local optimization problems are employed in the proposed model. Therefore, all subproblems can be disconnected in the current iteration and a sequence of optimizations is no longer required. To this end, a vector of coupling variables $\mathbf{Z}$ is created that can represent either target variables or response copies, and it contains the information exchanged with other subproblems. In this way, the target values from the upper level are transformed to $Z_{a a^{\prime}, s, t}^{(D N), n-1}$ and response values from the lower level are converted to $Z_{a a^{\prime}, s, t}^{\left(M G_{m}\right), n-1}$. Consequently, all subproblems will be in the same level and the values act as response copies in their penalty functions. However, the main issue in this procedure is the coordination of subproblems. Previous studies, e.g., Kargarian et al. [37], have proposed a central coordinator to provide each subproblem with target values in an optimal way. The objective of this central coordinator is to optimally minimize the summation of penalty functions of all subproblems. In this paper, similar to the study carried out by Kargarian et al. [38], the optimization problem of the central coordinator is distributed among all subproblems. Then, the distributed problem of the coordinator and a data exchange linking constraint are added to each subproblem as a constraint. Therefore, 
each subproblem becomes a bilevel optimization one as presented in the following abstract formulations:

$$
\begin{aligned}
\min _{\bar{x}^{(A)}, Z_{a a^{\prime}, s, t}^{(A)}} f^{(A)}\left(\bar{x}^{(A), n}\right)+\sum_{a a^{\prime} \in B_{n}^{(A)}} \sum_{s \in \mathbf{S}_{a a^{\prime}}^{(A)}} \sum_{t \in T} \\
\\
\left(\nu_{a a^{\prime}, s, t}^{(A), n} \times\left(Z_{a a^{\prime}, s, t}^{\left(C_{A}\right), n-1}-Z_{a a^{\prime}, s, t}^{(A), n}\right)\right. \\
\left.+\left\|\omega_{a a^{\prime}, s, t}^{(A), n} \circ\left(Z_{a a^{\prime}, s, t}^{\left(C_{A}\right), n-1}-Z_{a a^{\prime}, s, t}^{(A), n}\right)\right\|_{2}^{2}\right),
\end{aligned}
$$

s.t.:

$$
h^{(A)}\left(\bar{x}^{(A), n}, Z_{a a^{\prime}, s, t}^{(A)}\right)=0
$$

and:

$$
\begin{aligned}
& g^{(A)}\left(\bar{x}^{(A), n}, Z_{a a^{\prime}, s, t}^{(A)}\right) \geq 0 \\
& \arg \min _{Z_{a a^{\prime}, s, t}^{\left(C_{A}\right), n-1}} \sum_{a a^{\prime} \in B_{n}^{(A)}} \sum_{s \in \mathbf{S}_{a a^{\prime}}^{(A)}} \sum_{t \in T} I_{A}^{(C)} \\
& \left(\nu_{a a^{\prime}, s, t}^{(A), n-1} \times\left(Z_{a a^{\prime}, s, t}^{\left(C_{A}\right), n-1}-Z_{a a^{\prime}, s, t}^{(A), n-1}\right)\right. \\
& \left.+\left\|\omega_{a a^{\prime}, s, t}^{(A), n-1} \circ\left(Z_{a a^{\prime}, s, t}^{\left(C_{A}\right), n-1}-Z_{a a^{\prime}, s, t}^{(A), n-1}\right)\right\|_{2}^{2}\right), \\
& I_{A}^{(C)} \cdot Z_{a a^{\prime}, s, t}^{\left(C_{A}\right), n-1}=I_{A}^{(C)} \cdot Z_{a a^{\prime}, s, t}^{\left(C_{A^{\prime}}\right), n-1}\left(A \neq A^{\prime}\right),
\end{aligned}
$$

where $\bar{x}^{(A)}$ represents the local decision variables associated with subproblem $A ; Z_{a a^{\prime}, s, t}^{(A), n}$ is the coupling variable $s$ of shared nodes $a a^{\prime}$ at time $t$ in iteration $n ; f^{(A)}\left(\bar{x}^{(A)}\right)$ denotes the local operation cost of subproblem $A$ (i.e., $\left.\cos t_{\text {Tot }}^{A}\right) ; h^{(A)}$ and $g^{(A)}$ are abstract representations of equality and inequality constraints of subproblem $A$, respectively; $Z_{a a^{\prime}, s, t}^{\left(C_{A}\right), n-1}$ is the coordinated value of coupling variable $s$ of shared nodes $a a^{\prime}$ determined in the lower-level problem (i.e., the problem of the follower) at time $t$ in iteration $n-1$; $I_{A}^{(C)}$ is a binary incidence matrix that chooses the coordinated value of coupling variables of other agents associated with subproblem $A$; and $Z_{a a^{\prime}, s, t}^{\left(C_{A^{\prime}}\right), n-1}$ is the coordinated value of coupling variable $s$ of neighboring agent $A^{\prime}$ determined at time $t$ in iteration $n-1$. Constraint (34) demonstrates that agent $A$ has access only to the coupling variables of its direct neighbors $A^{\prime}$. Consequently, the privacy of information is met in each subproblem.

In order to transform the above bilevel optimization problem to a single-level model, the Karush-KuhnTucker (KKT) conditions are applied to the lower-level problem (Eqs. (33) and (34)) and then, these conditions are implemented in the constraints of the upper level.

Without loss of generality, we only consider exchanging a coupling variable $s$ between DN and $M G_{m}$ at time $t$. Thus, the lower-level problem (33) contains two penalty terms associated with the coupling variable $s$ shared by DN and $M G_{m}$ at time $t$, which can be formulated as follows:

$$
\begin{aligned}
\pi_{s, t}^{(c)}= & \left(\nu_{a a^{\prime}, s, t}^{(D N), n-1} \times\left(Z_{a a^{\prime}, s, t}^{\left(C_{D N}\right), n-1}-Z_{a a^{\prime}, s, t}^{(D N), n-1}\right)\right. \\
& \left.+\left\|\omega_{a a^{\prime}, s, t}^{(D N), n-1} \circ\left(Z_{a a^{\prime}, s, t}^{\left(C_{D N}\right), n-1}-Z_{a a^{\prime}, s, t}^{(D N), n-1}\right)\right\|_{2}^{2}\right) \\
& +\left(\nu_{a a^{\prime}, s, t}^{\left(M G_{m}\right), n-1} \times\left(Z_{a a^{\prime}, s, t}^{\left(C_{M G}\right), n-1}-Z_{a a^{\prime}, s, t}^{\left(M G_{m}\right), n-1}\right)\right. \\
& +\| \omega_{a a^{\prime}, s, t}^{\left(M G_{m}\right), n-1} \circ\left(Z_{a a^{\prime}, s, t}^{\left(C_{M G_{m}}\right), n-1}\right. \\
& \left.\left.-Z_{a a^{\prime}, s, t}^{\left(M G_{m}\right), n-1}\right) \|_{2}^{2}\right) .
\end{aligned}
$$

Based on the lower-level constraint (34), we have $Z_{a a^{\prime}, s, t}^{\left(C_{D N}\right), n-1}=Z_{a a^{\prime}, s, t}^{\left(C_{M G}\right), n-1}$. Therefore, by replac$\operatorname{ing} Z_{a a^{\prime}, s, t}^{\left(C_{M G_{m}}\right), n-1}$ with $Z_{a a^{\prime}, s, t}^{\left(C_{D N}\right), n-1}$ and taking partial derivative of $\pi_{s, t}^{(c)}$ with respect to $Z_{a a^{\prime}, s, t}^{\left(C_{D N}\right), n-1}$, we have:

$$
\begin{gathered}
\partial \pi_{s, t}^{(c)} / \partial Z_{a a^{\prime}, s, t}^{\left(C_{D N}\right), n-1}=\nu_{a a^{\prime}, s, t}^{(D N), n-1}+2 \omega_{a a^{\prime}, s, t}^{(D N), n-1} \\
Z_{a a^{\prime}, s, t}^{(D N), n-1}-2 \omega_{a a^{\prime}, s, t}^{(D N), n-1} Z_{a a^{\prime}, s, t}^{(D N), n-1} \\
+\nu_{a a^{\prime}, s, t}^{\left(M G_{m}\right), n-1}+2 \omega_{a a^{\prime}, s, t}^{\left(M G_{m}\right), n-1} Z_{a a^{\prime}, s, t}^{\left(C_{D N}\right), n-1} \\
-2 \omega_{a a^{\prime}, s, t}^{\left(M G_{m}\right), n-1} Z_{a a^{\prime}, s, t}^{\left(M G_{m}\right), n-1}=0
\end{gathered}
$$

Thus, $Z_{a a^{\prime}, s, t}^{\left(C_{D N}\right), n-1}$ can be calculated by Eq. (37) as shown in Box II. $Z_{a a^{\prime}, s, t}^{\left(C_{M G}\right), n-1}$ is also calculated in the same way.

$$
Z_{a a^{\prime}, s, t}^{\left(C_{D N}\right), n-1}=\frac{2 \omega_{a a^{\prime}, s, t}^{(D N), n-1} Z_{a a^{\prime}, s, t}^{(D N), n-1}+2 \omega_{a a^{\prime}, s, t}^{\left(M G_{m}\right), n-1} Z_{a a^{\prime}, s, t}^{\left(M G_{m}\right), n-1}-\nu_{a a^{\prime}, s, t}^{(D N), n-1}-\nu_{a a^{\prime}, s, t}^{\left(M G_{m}\right), n-1}}{2 \omega_{a a^{\prime}, s, t}^{(D N), n-1}+2 \omega_{a a^{\prime}, s, t}^{\left(M G_{m}\right), n-1}}
$$


Now, the lower-level optimization problem, i.e., Eqs. (33) and (34), can be replaced with its KKT conditions in the upper-level problem and the resulting formulation for subproblem $A$ can be rewritten as follows:

$$
\begin{aligned}
\min _{\bar{x}^{(A)}, Z_{a a^{\prime}, s, t}^{(A)}} & f^{(A)}\left(\bar{x}^{(A), n}\right)+\sum_{a a^{\prime} \in B_{n}^{(A)}} \sum_{s \in \mathbf{S}_{a a^{\prime}}^{(A)}} \sum_{t \in T} \\
& \left(\nu_{a a^{\prime}, s, t}^{(A), n} \times\left(Z_{a a^{\prime}, s, t}^{\left(C_{A}\right), n-1}-Z_{a a^{\prime}, s, t}^{(A), n}\right)\right. \\
& \left.+\left\|\omega_{a a^{\prime}, s, t^{\circ}}^{(A), n}\left(Z_{a a^{\prime}, s, t}^{\left(C_{A}\right), n-1}-Z_{a a^{\prime}, s, t}^{(A), n}\right)\right\|_{2}^{2}\right),
\end{aligned}
$$

s.t.:

$h^{(A)}\left(\bar{x}^{(A), n}, Z_{a a^{\prime}, s, t}^{(A)}\right)=0, \quad g^{(A)}\left(\bar{x}^{(A), n}, Z_{a a^{\prime}, s, t}^{(A)}\right) \geq 0$.

Eq. (40) is shown in Box III.

In iteration $n$, subproblem $A$ relies on its local information $\bar{x}^{(A), n}$ as well as $Z_{a a^{\prime}, s, t}^{\left(C_{A}\right), n-1}$, wherein $Z_{a a^{\prime}, s, t}^{\left(C_{A}\right), n-1}$ is determined via coupling variables between this agent and its direct neighboring agents in iteration $n-1$. Also, the Lagrange multiplier $\nu_{a a^{\prime}, s, t}^{(A), n}$ and the penalty $\omega_{a a^{\prime}, s, t}^{(A), n}$ are updated as:

$$
\begin{aligned}
& \nu_{a a^{\prime}, s, t}^{(A), n}= \nu_{a a^{\prime}, s, t}^{(A), n-1}+2\left(\omega_{a a^{\prime}, s, t}^{(A), n-1}\right)^{2} \\
&\left(Z_{a a^{\prime}, s, t}^{\left(C_{A}\right), n-1}-Z_{a a^{\prime}, s, t}^{(A), n-1}\right), \\
& \omega_{a a^{\prime}, s, t}^{(A), n}=\gamma \cdot \omega_{a a^{\prime}, s, t}^{(A), n-1},
\end{aligned}
$$

where $\gamma \geq 1$ is necessary for improving the convergence performance. We recommend a value close to 1 for most cases. As in iteration $n$, each subproblem requires the values of the coupling variables in its connected neighbors in iteration $n-1$ so that both DN and MGs problems can be solved in a decentralized and parallel manner in iteration $n$. Also, the convergence of the whole network is determined based on the consistency between the current pair of target and response values as formulated in Eq. (43):

$$
\mathbf{C}_{a a^{\prime}, t}^{(A), n}=\mathbf{Z}_{a a^{\prime}, t}^{\left(C_{A}\right), n}-\mathbf{Z}_{a a^{\prime}, t}^{(A), n} .
$$

To summarize, the parallel solution procedure based

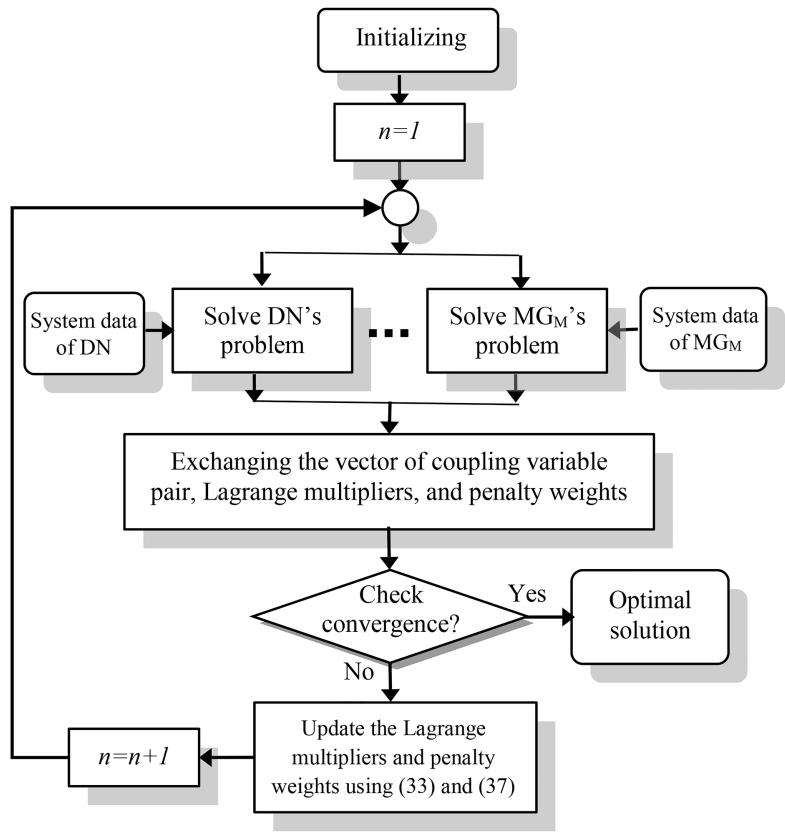

Figure 4. Flowchart of the proposed non-hierarchical ATC.

on the proposed non-hierarchical ATC model is presented by the following algorithm. Also, the flowchart associated with the proposed procedure is depicted in Figure 4.

Step 1. Set the iteration index $n=1$; select initial values for $\nu_{a a^{\prime}, s, t}^{(A), 1}, \omega_{a a^{\prime}, s, t}^{(A), 1}$, and $Z_{a a^{\prime}, s, t}^{\left(C_{A}\right), 0}$; and set $\gamma$ and the convergence tolerances $\varepsilon>0$;

Step 2. Solve the local optimization problems (38)(40) for both DN and MGs in parallel and determine the optimal value for the coupling variables $Z_{a a^{\prime}, s, t}^{(A), n}$;

Step 3. Exchange the vector of coupling variable pair $\left(\mathbf{Z}_{a a^{\prime}, t}^{(D N), n}, \mathbf{Z}_{a a^{\prime}, t}^{\left(M G_{m}\right), n}\right)$, Lagrange multipliers $\nu_{a a^{\prime}, s, t}^{(A), 1}$, and penalty $\omega_{a a^{\prime}, s, t}^{(A), 1}$ among neighboring agents;

Step 4. Compute the vector of consistency between the current pair of target and response values. If $\left\|\mathbf{C}_{a a^{\prime}, t}^{(A), n}\right\|_{\max } \leq \varepsilon$, the procedure stops and the optimal decision variables are obtained; otherwise, go to Step 5;

Step 5. Update the Lagrange multipliers and penalty weights using Eqs. (41) and (42), respectively;

Step 6. Set $n=n+1$ and go to Step 2 .

$$
Z_{a a^{\prime}, s, t}^{\left(C_{A}\right), n-1}=\frac{2 \omega_{a a^{\prime}, s, t}^{(A), n-1} Z_{a a^{\prime}, s, t}^{(A), n-1}+2 \omega_{a a^{\prime}, s, t}^{\left(A^{\prime}\right), n-1} Z_{a a^{\prime}, s, t}^{\left(A^{\prime}\right), n-1}-\nu_{a a^{\prime}, s, t}^{(A), n-1}-\nu_{a a^{\prime}, s, t}^{\left(A^{\prime}\right), n-1}}{2 \omega_{a a^{\prime}, s, t}^{(A), n-1}+2 \omega_{a a^{\prime}, s, t}^{\left(A^{\prime}\right), n-1}} \quad \forall A^{\prime} \in N^{(A)}
$$




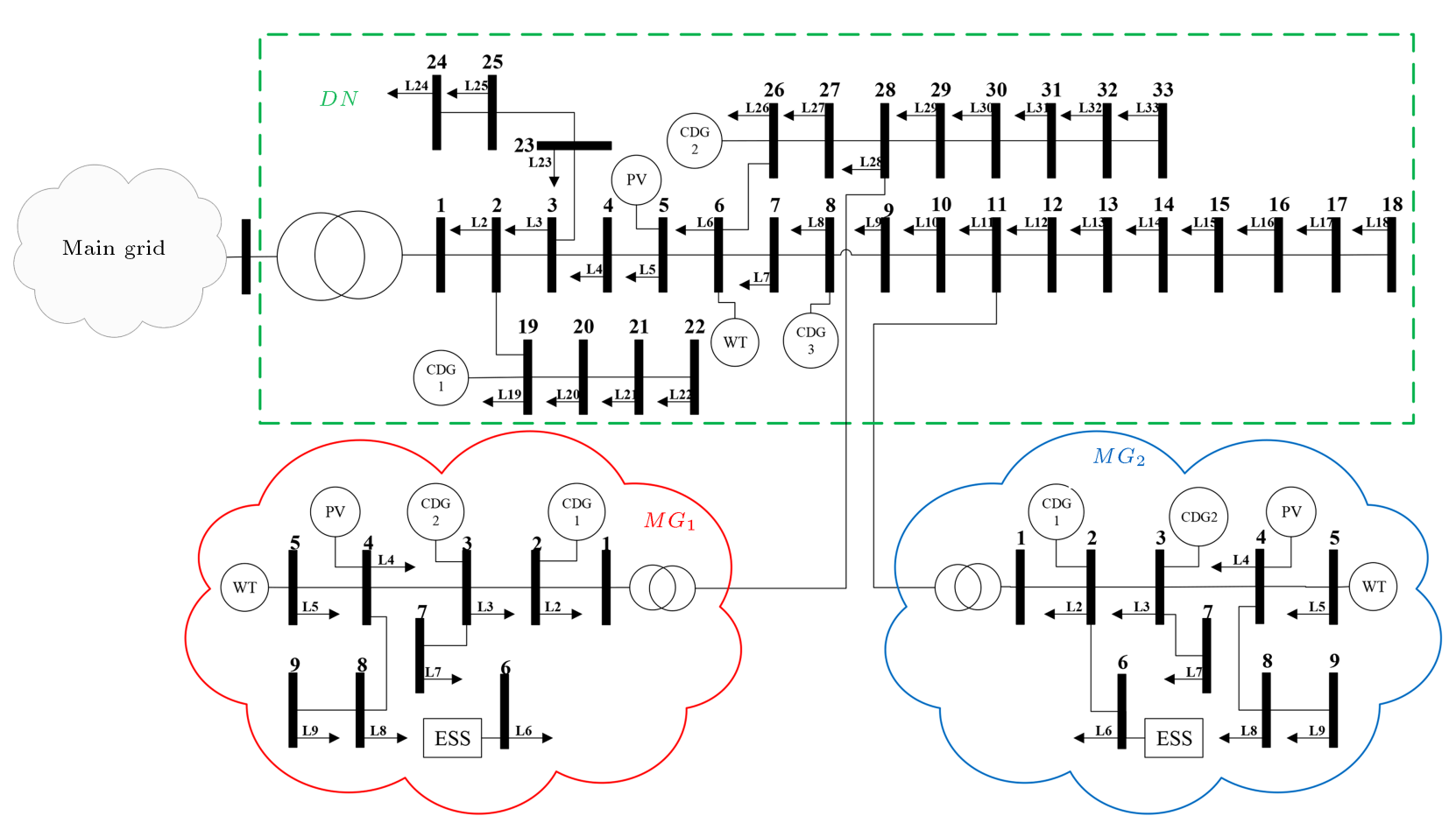

Figure 5. Modified IEEE 33-bus test system with two MGs.

Table 1. CDGs data for both DN and MGs.

\begin{tabular}{|c|c|c|c|c|c|c|c|c|}
\hline & CDG & $\begin{array}{l}\underline{P}_{i, t}^{D G} \\
(\mathrm{~kW})\end{array}$ & $\begin{array}{l}\overline{\boldsymbol{P}}_{i, t}^{D G} \\
(\mathrm{~kW})\end{array}$ & $\begin{array}{c}a_{i, t}^{D G} \\
\left(\$ / \mathbf{k W h}^{2}\right)\end{array}$ & $\begin{array}{c}b_{i, t}^{D G} \\
\left(\$ / \mathbf{k W h}^{2}\right)\end{array}$ & $\begin{array}{c}c_{i, t}^{D G} \\
(\$ / \mathbf{h})\end{array}$ & $\begin{array}{c}C r_{i, t}^{u p} \\
(\$ / \mathbf{k W h})\end{array}$ & $\begin{array}{c}C r_{i, t}^{d n} \\
(\$ / \mathbf{k W h})\end{array}$ \\
\hline \multirow{3}{*}{ DN } & CDG1 & 100 & 2000 & 0.0001 & 0.27 & 0 & 0.060 & 0.060 \\
\hline & CDG2 & 100 & 2000 & 0.0004 & 0.40 & 0 & 0.050 & 0.050 \\
\hline & CDG3 & 100 & 2000 & 0.0003 & 0.55 & 0 & 0.040 & 0.040 \\
\hline \multirow{2}{*}{$M G_{1}$ and $M G_{2}$} & CDG1 & 60 & 1000 & 0.0004 & 0.47 & 0 & 0.077 & 0.077 \\
\hline & CDG2 & 40 & 700 & 0.0006 & 0.58 & 0 & 0.044 & 0.044 \\
\hline
\end{tabular}

Table 2. The data of energy storage systems.

\begin{tabular}{ccccccc}
\hline & $\overline{\boldsymbol{P}}_{i, t}^{S C}(\mathrm{~kW})$ & $\underline{\boldsymbol{E}}_{i, t}^{S}(\mathrm{kWh})$ & $\overline{\boldsymbol{E}}_{i, t}^{S}(\mathbf{k W h})$ & $C_{i, t}^{S C}(\$ / \mathbf{k W h})$ & $\boldsymbol{C}_{i, t}^{S D}(\$ / \mathbf{k W h})$ & $\boldsymbol{\eta}_{i, t}^{S C(A)} / \boldsymbol{\eta}_{i, t}^{S D(A)}$ \\
\hline $\boldsymbol{M} \boldsymbol{G}_{\mathbf{1}}$ and $\boldsymbol{M} \boldsymbol{G}_{\mathbf{2}}$ & 40 & 100 & 400 & 0.4 & 0.6 & 0.96 \\
\hline
\end{tabular}

\section{Simulation results}

The proposed ATC-based decentralized energy trading algorithm is applied to the modified IEEE 33-bus system [39]. The computations for all tests are conducted using YALMIP toolbox [40] and ILOG CPLEX $12.5 \mathrm{~s}$ [41] MIQCP solver on a $2.30-\mathrm{GHz}$ intel Core i5 CPU personal computer with 4 GB of RAM memory.

The modified IEEE 33-bus test system has 3 CDGs, one wind unit, and one PV, as shown in Figure 5. In addition, the system is comprised of two MGs named $M G_{1}$ and $M G_{2}$, which are respectively connected to the DN through nodes 11 and 28. Both $M G_{1}$ and $M G_{2}$ are identical and 9-bus systems with
2 CDGs, one wind unit, one PV, and one ESS. The data for the CDGs and ESSs are given in Tables 1 and 2 , respectively. Daily load profile, wholesale energy market price, and the forecast production of both wind and PV units for DN and MGs are shown in Figure 6. $\bar{R}_{t}^{E E N S}$ and $\bar{R}_{t}^{E R C}$ for DN and MGs are $10 \%$ of the total demand and renewable generation in each time interval, respectively. Moreover, the associated costs of EENS and ERC are respectively set to 5 times and 2 times the wholesale energy price at each hour. The initial values for all coupling variables and Lagrange multiplier variables are set to zero and the values of the penalty weights are initialized with the value of 1. Also, $\gamma=1.05$ and the convergence threshold is 


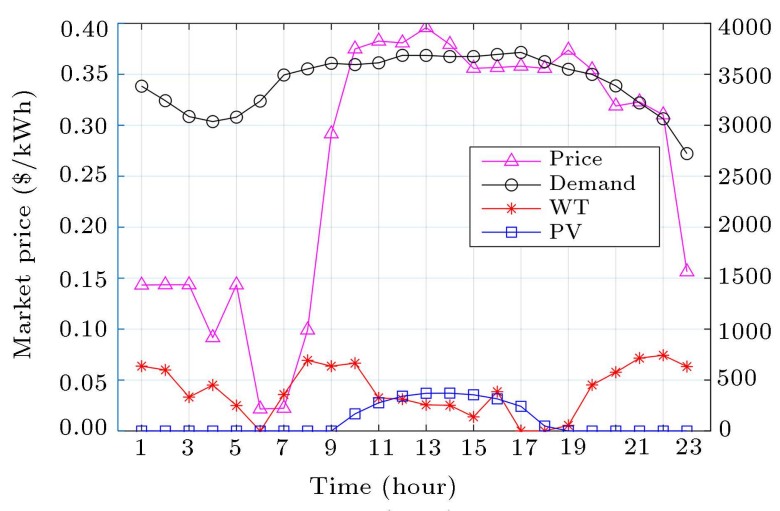

(a) DN

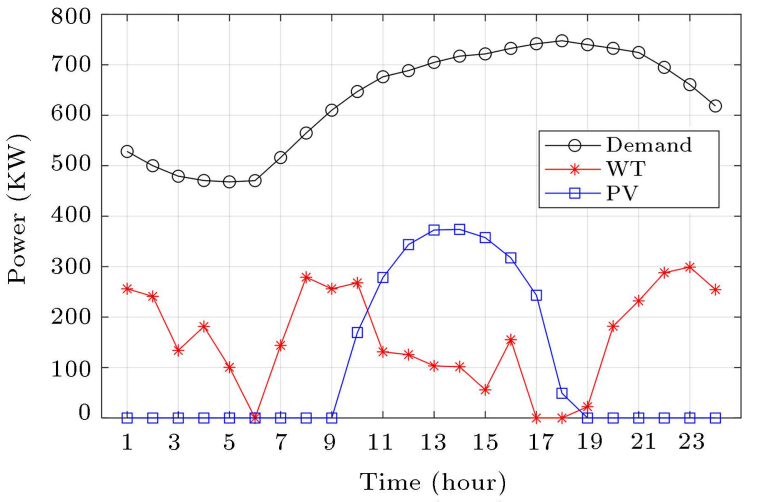

(b) $M G_{1} \& M G_{2}$

Figure 6. Daily load profile, renewable generation forecast, and wholesale market price of the system.

$\varepsilon=0.001$. Voltage of substation node is set to 1 p.u. and voltages of other nodes are constrained to 0.9 p.u. up to 1.1 p.u..

Based on the above information, the proposed model is preformed and the obtained results are described in the following. The daily resource scheduling for DN, $M G_{1}$, and $M G_{2}$ is shown in Figure 7 . As it can be seen, all CDGs in DN, $M G_{1}$, and $M G_{2}$ are committed throughout the operating horizon given either their low operating cost or up/down reserve for dealing with real-time uncertainties. Since the wholesale energy price is relatively lower than operating cost of the CDGs, DN decides to import power from the main grid and sell a portion of it to $M G_{1}$ and $M G_{2}$ during hours 1-6 and 18-24. For the rest of the day, $M G_{1}$ and $M G_{2}$ export their energy surplus to DN. As a result, both DN and MGs, besides meeting their local demands, optimally provide part of the required demand of each other through the tie-lines. Moreover, both $E S S_{1}$ and $E S S_{2}$ are being charged during offpeak hours and deliver their stored energy to MGs when the demand is high. It should be noted that the charging of $E S S_{1}$ and $E S S_{2}$ at hours 23 and 24 is due to their state of charge limitations. By comparing the total local generation with total local demand as well as the imported/exported power in Figure 7, it is clearly evident that the power balance in the proposed model is accurately guaranteed throughout the operating day. It is worth mentioning although CDGs in both DN and MGs can provide more power, their remaining capacities are considered as reserves to manage uncertainty of renewables and demand in real time.

In order to validate efficiency and optimality of the proposed method, the test case results are compared with the solutions of centralized and hierarchical approaches via identical parameters. Note that in the case of the centralized approach, the whole information is available to a central operator, which solves the scheduling problem for both DN and MGs. Recall that the centralized approach is questionable for implementation in the future distribution networks due to many reasons described in Section 1 and this comparison is only to validate the optimality of the results obtained by the proposed method. According to Table 3, both hierarchical ATC and the proposed non-hierarchical ATC methods can converge to the

Table 3. Comparison of centralized and decentralized approaches.

\begin{tabular}{ccccc}
\hline Method & & Centralized & Hierarchical ATC & Non-hierarchical ATC \\
\hline \multirow{2}{*}{ Total generation cost (\$) } & $\mathrm{DN}$ & 25068.502 & 25066.119 & 25061.257 \\
& $M G_{1}$ & 4996.0898 & 4995.986 & 4998.547 \\
& $M G_{2}$ & 5387.2414 & 5388.599 & 5390.470 \\
Total reserve cost (\$) & $\mathrm{DN}$ & 5422.854 & 5422.620 & 5422.143 \\
& $M G_{1}$ & 947.468 & 947.462 & 947.680 \\
& $M G_{2}$ & 953.734 & 953.705 & 953.654 \\
Total risk cost (\$) & & & & 619.981 \\
& $\mathrm{DN}$ & 618.925 & 619.260 & 160.479 \\
Total operation cost $(\$)$ & $M G_{1}$ & 160.800 & 160.827 & 138.795 \\
& $M G_{2}$ & 138.772 & 138.772 & 43693.006 \\
& & & & $($ gap $0.031 \%)$ \\
\hline
\end{tabular}




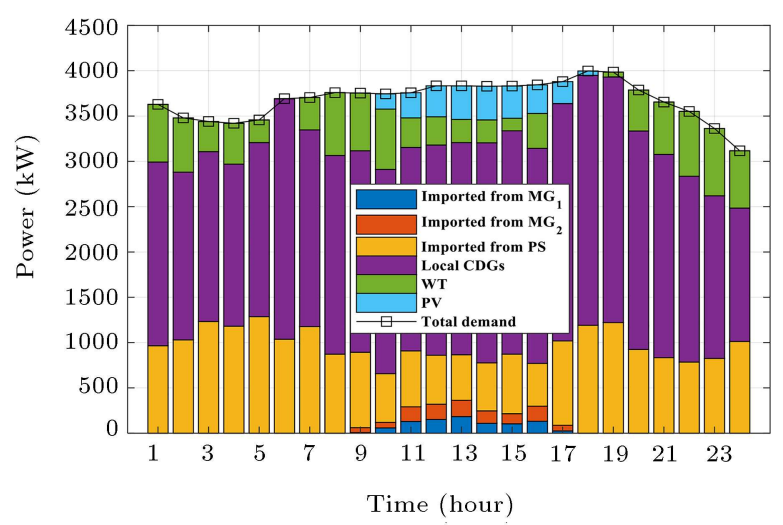

(a) Total local generation plus total imported power

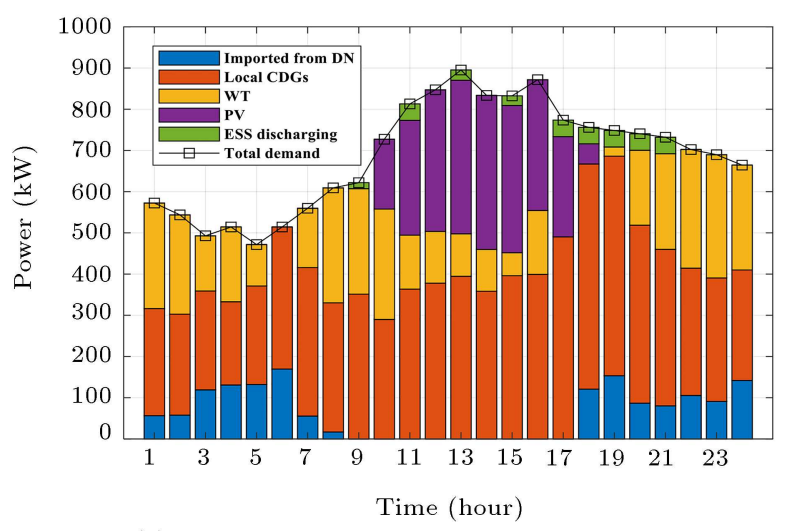

(c) Total local generation plus total imported power

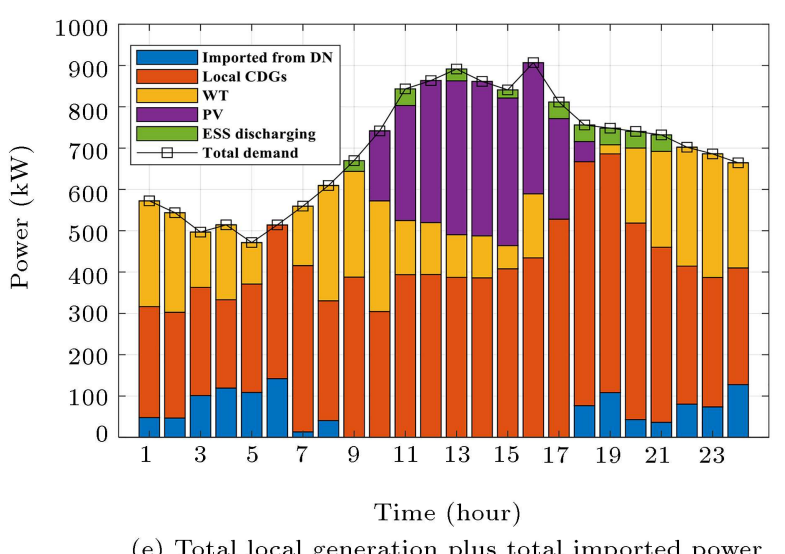

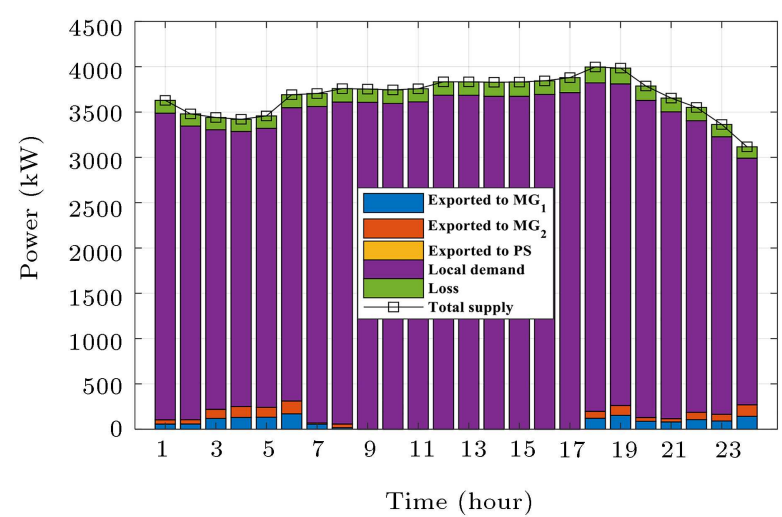

(b) Total local demand plus total exported power

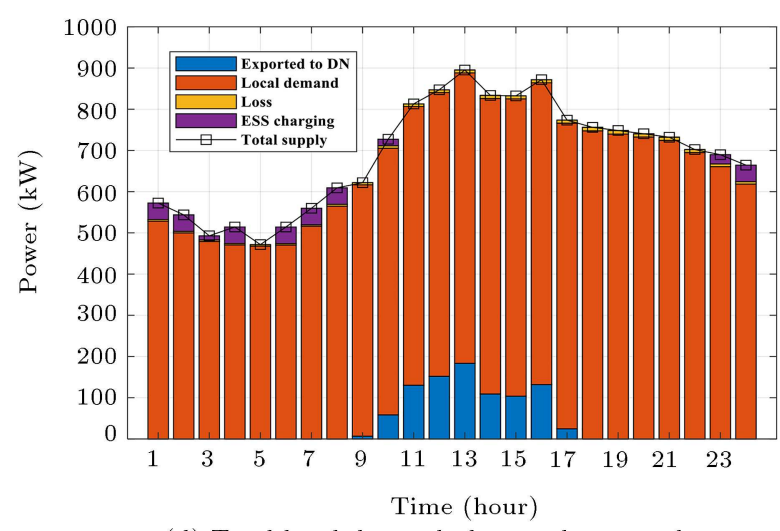

(d) Total local demand plus total exported power

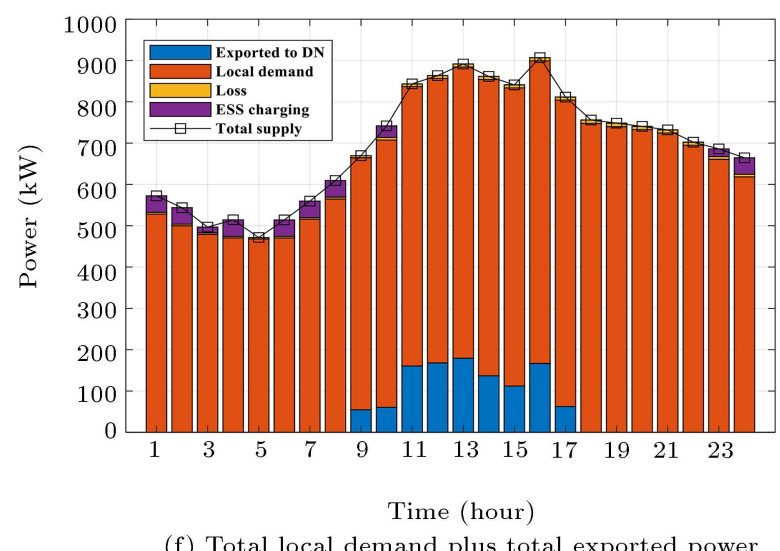

(f) Total local demand plus total exported power

Figure 7. Daily scheduling: (a) and (b) DN; (c) and (d) $M G_{1}$; and (e) and (f) $M G_{2}$.

optimal solution since their objective values are slightly different from the centralized one.

To demonstrate the superiorities of the proposed non-hierarchical model over the hierarchical ATC, the convergence criterion, i.e.:

$$
\left\|\mathbf{C}_{a a^{\prime}, t}^{(A), n}\right\|_{\max }
$$

is depicted in Figure 8. As it can be seen, the hierarchical ATC method converges after 109 iterations, while the proposed method finds the optimal solution after 75 iterations. It is worth noting that the hierarchical ATC is a sequential approach, while the proposed method is a parallel one. Thus, the proposed method requires much less computing time with fewer iterations.

Also, we consider random attacks on the coupling values received from MGs in iterations 32-37. As it is shown in Figure 8, the proposed method converges after 75 iterations, which is the same as the base case with no attack. Therefore, the proposed algorithm is reasonably resistant to cyber-attacks and provides the optimal solution. 


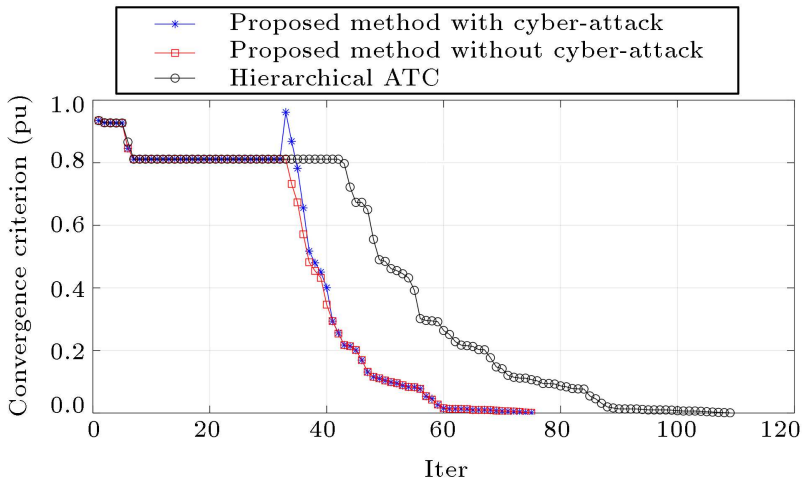

Figure 8. Convergence criterion.

Finally, an analysis based on Monte Carlo simulation was performed to examine robustness of the solution obtained by the proposed method in dealing with the real-time uncertainties. The day-ahead scheduling solutions were evaluated via 1000 scenarios representing different realizations of net demand uncertainty. The results are studied in 4 aspects, namely insecure scenarios, EENS, ERC, and Expected Dispatch Cost (EDC). Insecure scenarios represent those in which the given solutions fail to satisfy the operational security constraints in at least one timeinterval. In this study, the security of the system has been compromised in only 7 scenarios. In other scenarios, CDGs have successfully responded to the real-time uncertainties. In addition, EENS and ERC are 0.003 $\mathrm{kWh}$ and $1.01 \mathrm{kWh}$, respectively, which are very trivial in comparison with the total net demand of the entire network. The EDC of the whole network is $\$ 41496.73$. This value is almost close to the total operation cost (generation cost plus reserve cost) given in Table 3 and shows economic efficiency of the solution.

\section{Conclusions}

The objective of this paper was to develop a fully decentralized decision making algorithm for collaborative scheduling of ADN with MMGs. The proposed algorithm was based on ATC technique in which the scheduling optimization problem of DN was located in the upper level of the hierarchy, while the local optimization problems of MGs were considered in the lower level. To provide a fully parallelized solution procedure, a non-hierarchical ATC algorithm having no central coordinator was developed. The proposed model was an MISOCP problem that considered the exact model of MGs network using the AC optimal power flow, while only limited information was exchanged with DN. The case studies of a modified IEEE 33-bus distribution test system showed the accuracy and convergence of the proposed method. Although both hierarchical and the proposed non-hierarchical ATC algorithms provided reasonable outcomes, the proposed method generally

converged in a lower number of iterations and required much less time thanks to parallelization. In addition, the results demonstrated that the proposed algorithm was potentially resistant to cyber-attacks. Also, the proposed procedure solutions could well guarantee security of the system against multiple source-load uncertainties.

\section{Acknowledgment}

The authors would like to acknowledge the financial support of Lorestan University for the research under Grant Number 985011399.

\section{Nomenclature}

\section{Sets and indices}

$n$

$a, a^{\prime}$

$i, j, k$

$t, h$

$s$

$m$

$A$

$T$

$\operatorname{Sub}(D N)$

$D G(A)$

$\operatorname{ESS}(A)$

$B_{n}^{(A)}$

$\boldsymbol{S}_{a a^{\prime}}^{(A)}$

$N^{(M G)}$

$N^{(A)}$

\section{Parameters}

$C_{i, t}^{S u b}$

$a_{i, t}^{D G}, b_{i, t}^{D G}, c_{i, t}^{D G}$

$C r_{i, t}^{u p} / C r_{i, t}^{d n}$

$C_{i, t}^{S C} / C_{i, t}^{S D}$

$C_{t}^{E R C}$

$C_{t}^{E E N S}$

$\underline{P}_{i}^{D G} / \bar{P}_{i}^{D G}$
Index of iterations

Indices of border nodes

Indices of nodes

Indices of time/hour

Index of coupling variables

Index of MGs

Index of agents

Set of time intervals

Set of substations connecting DN to the main grid

Set of CDGs in agent $A$

Set of ESSs in agent $A$

Set of border nodes of agent $A$

Set of coupling variables of border nodes $a a^{\prime}$ shared by agent $A$

Set of MGs

Set of neighboring agents of agent A

Energy price of purchasing power from the main grid

Cost coefficients of power generation by a CDG

Up/down scheduled reserve cost of a CDG

Charging/discharging cost of an ESS

Cost of expected renewable energy curtailment

Cost of expected energy not supplied

Minimum/maximum active power output of a CDG 
$\underline{Q}_{i}^{D G(c)} / \bar{Q}_{i}^{D G}$

$\bar{S}_{i}^{D G}$

$\bar{R}_{i}^{u p} / \bar{R}_{i}^{d n}$

$R U_{i}^{D G} / R D_{i}^{D G}$

$D T_{i} / U T_{i}$

$T_{i}^{\mathrm{ON}} / T_{i}^{\mathrm{OFF}}$

\section{$\Delta T$}

$\bar{P}_{i,}^{S C} / \bar{P}_{i}^{S D}$

$\eta_{i}^{S C} / \eta_{i, t}^{S D}$

$\bar{E}_{i}^{S} / \underline{E}_{i}^{S}$

$D_{t}^{n e t(A)}$

$\underline{D}_{t}^{n e t(A)}$

$\bar{D}_{t}^{n e t(A)}$

$f_{\text {net }, t}^{A}(x)$

$P_{i, t}^{L} / Q_{i, t}^{L}$

$P_{i, t}^{W}$

$P_{i, t}^{P V}$

$\bar{R}_{t}^{E E N S(A)}$

$\bar{R}_{t}^{R C(A)}$

$r_{i j} / x_{i j}$

$g_{i} / b_{i}$

$\bar{i}_{i}$

$\bar{v}_{i} / \underline{v}_{i}$

$\gamma$

$\nu_{a a^{\prime}, s, t}^{(A), n} / \omega_{a a^{\prime}, s, t}^{(A), n}$

$Z_{a a^{\prime}, s, t}^{\left(C_{A}\right), n-1}$
Minimum/maximum reactive power limit of a CDG

Maximum apparent power output of a $\mathrm{CDG}$

Maximum up/down scheduled reserve of a CDG

Maximum up/down ramp rate of a CDG

Minimum down/up time of a CGD

The number of initial periods during which a CDG must be on/off

Duration of time interval

Maximum charging/discharging power of an ESS

Charging/discharging efficiency of an ESS

Maximum/minimum of stored energy in an ESS

Expected net demand in time $t$ by agent $A$

Minimum net demand in time $t$ by agent $A$

Maximum net demand in time $t$ by agent $A$

Probability of net demand in time $t$ for agent $A$

Forecasted active/reactive demand at node $i$ in time $t$

Forecasted wind power generation at node $i$ in time $t$

Forecasted $\mathrm{PV}$ generation at node $i$ in time $t$

Maximum allowable EENS in time $t$ for agent $A$

Maximum allowable ERC in time $t$ for agent $A$

Resistance/reactance of line $i j$

Conductance/suseptance of node

Minimum current of node

Minimum/maximum voltage of node

Tuning parameter

Lagrange multiplier/penalty weight associated with the coupling variable $s$ of shared nodes $a a^{\prime}$ in time $t$ in iteration $n$

Coordinated value of the coupling variable $s$ of agent $A$ determined in time $t$ in iteration $n-1$

\section{Variables}

$P_{i, t}^{D G(A)} / Q_{i, t}^{D G(A)}$ Active/reactive power output of a CDG

$R_{i, t}^{u p(A)} / R_{i, t}^{d n(A)} \quad \mathrm{Up} /$ down scheduled reserve of a CDG

$P_{i, t}^{S C(A)} / P_{i, t}^{S D(A)}$ Charging/discharging power of an ESS

$P_{i, t}^{S u b(D N)} /$

$Q_{i, t}^{i, t} \operatorname{Sub(DN)}$

Active/reactive power transmitted

$R_{t}^{E R C(A)}$ through substation

$R_{t}^{E E N S(A)}$

$E_{i, t}^{S(A)}$

$u_{i, t}^{D G(A)}$

ERC in time $t$ for agent $A$

EENS in time $t$ for agent $A$

Energy stored in an ESS

$u_{i, t}^{S C(A)} / u_{i, t}^{S D(A)}$

Binary variable indicating the status of a CDG

Binary variable indicating charging/discharging status of an ESS

$P_{i j, t}^{(A)} / Q_{i j, t}^{(A)}$

Active/reactive power of line $i j$ in time $t$ for agent $A$

$V_{i, t}^{(A)}$

$I_{i k, t}^{(A)}$

$Z_{a a^{\prime}, s, t}^{(A), n}$

Squared voltage magnitude of node $i$ in time $t$ for agent $A$

Squared current of line $i j$ in time $t$ for agent $A$

Coupling variable $s$ of shared nodes $a a^{\prime}$ in time $t$ in iteration $n$

\section{References}

1. Fotuhi-Friuzabad, M., Safdarian, A., and MoeiniAghtaie, M., et al. "Upcoming challenges of future electric power systems: sustainability and resiliency", Scientia Iranica, 23(4), pp. 1565-1577 (2016).

2. Shahidehpour, M. and Fotuhi-Friuzabad, M. "Grid modernization for enhancing the resilience, reliability, economics, sustainability, and security of electricity grid in an uncertain environment", Scientia Iranica, 23(16), pp. 2862-2873 (2016).

3. Lu, W., Liu, M., Lin S., et al. "Incremental-oriented ADMM for distributed optimal power flow with discrete variables in distribution networks", IEEE Trans. Smart Grid, 10(6), pp. 6320-6331 (Nov. 2019). DOI: 10.1109/TSG.2019.2902255

4. Ross, M., Abbey, C., Bouffard, F., et al. "Multiobjective optimization dispatch for microgrids with a high penetration of renewable generation" , IEEE Trans. Sustain. Energy, 6(4), pp. 1306-1314 (2015).

5. Fathi M. and Bevrani, H. "Statistical cooperative power dispatching in interconnected microgrids", IEEE Trans. Sustain. Energy, 4(3), pp. 586-593 (2013).

6. Ouammi, A., Dagdougui, H., Dessaint L., et al. "Coordinated model predictive-based power flows control 
in a cooperative network of smart microgrids", IEEE Trans. Smart Grid, 6(5), pp. 2233-2244 (2015).

7. Haddadian, H. and Noroozian, R. "Multi-microgrids approach for design and operation of future distribution networks based on novel technical indices", Appl. Energy, 185, pp. 650-663 (2017).

8. Haddadian, H. and Noroozian, R. "Multi-Microgridbased operation of active distribution networks considering demand response programs", IEEE Trans. Sustain. Energy, 10(4), pp. 1804-1812 (Oct. 2019). DOI:10.1109/TSTE.2018.2873206

9. Zhang, B., Li Q., Wang, L., and Feng, W. "Robust optimization for energy transactions in multi-microgrids under uncertainty" , Appl. Energy, 217, pp. 346-360 (2018).

10. Alam, M.N., Chakrabarti, S., and Ghosh, A. "Networked microgrids: State-of-the-art and future perspectives", IEEE Trans. Ind. Inform., 15(3), pp. 12381250 (2019).

11. Toutounchi, A.N., Seyedshenava, S., Contreras J., et al. "A stochastic bilevel model to manage active distribution networks with multi-microgrids", IEEE Syst. J., 13(4), pp. 4190-4199 (Dec. 2019). DOI: 10.1109/JSYST.2018.2890062

12. Wang, Z., Chen, B., Wang, J., et al. "Coordinated energy management of networked microgrids in distribution systems", IEEE Trans. Smart Grid, 6(1), pp. 45-53 (2015).

13. Minciardiand, R. and Robba, M. "A bilevel approach for the stochastic optimal operation of interconnected microgrids", IEEE Trans. Autom. Sci. Eng., 14(2), pp. 482-493 (2017).

14. Wang, H. and Huang, J. "Incentivizing energy trading for interconnected microgrids" , IEEE Trans. Smart Grid, 9(4), pp. 2647-2657 (2018).

15. Park, S., Lee, J., Bae, S., et al. "Contribution-based energy-trading mechanism in microgrids for future smart grid: A game theoretic approach", IEEE Trans. Ind. Electron., 63(7), pp. 4255-4265 (2016).

16. Jadhav, A.M. and Patne, N.R. "Priority-based energy scheduling in a smart distributed network with multiple microgrids", IEEE Trans. Ind. Inform., 13(6), pp. 3134-3143 (2017).

17. Du, Y., Wang, Z., Liu, G., et al. "A cooperative game approach for coordinating multi-microgrid operation within distribution systems", Appl. Energy, 222, pp. 383-395 (2018).

18. Mei, J., Chen, C., Wang, J., et al. "Coalitional game theory based local power exchange algorithm for networked microgrids", Appl. Energy, 239, pp. 133-141 (2019).

19. Liu, Y., Guo, L., and Wang, C. "A robust operationbased scheduling optimization for smart distribution networks with multi-microgrids", Appl. Energy, 228, pp. 130-140 (2018).
20. Nunna, H. and Doolla, S. "Multiagent-based distributed-energy-resource management for intelligent microgrids", IEEE Trans. Industr. Electron., 60(4), pp. 1678-1687 (2013).

21. Rahman, M.S. and Oo, A.M.T. "Distributed multiagent based coordinated power management and control strategy for microgrids with distributed energy resources", Energy Convers. Manage., 139, pp. 20-32 (2017).

22. Ju, L., Zhang, Q., Tan, Z., et al. "Multi-agentsystem-based coupling control optimization model for micro-grid group intelligent scheduling considering autonomy-cooperative operation strategy", Energy, 157, pp. 1035-1052 (2018).

23. Kou, P., Liang, D., and Gao L. "Distributed EMPC of multiple microgrids for coordinated stochastic energy management", Appl. Energy, 185, pp. 939-952, (2017).

24. Holjevac, N., Capuder, T., Zhang, N., et al. "Corrective receding horizon scheduling of flexible distributed multi-energy microgrids", Appl. Energy, 207, pp. 176194 (2017).

25. Wang, H. and Huang, J. "Incentivizing energy trading for interconnected microgrids", IEEE Trans. Smart Grid, 7(6), pp. 2647-2657 (2018).

26. Gao, H., Liu, J., Wang, L., et al. "Decentralized energy management for networked microgrids in future distribution systems", IEEE Trans. Power Syst., 33(4), pp. 3599-3610 (2018).

27. Feng, C., Wen, F., Zhang, L., et al. "Decentralized energy management of networked microgrid based on alternating-direction multiplier method", Energies, 11(10), p. 2555 (2018).

28. Mohiti, M., Monsef, H., Anvari-Moghaddam, A., et al. "A decentralized robust model for optimal operation of distribution companies with private microgrids", Int. J. Elect. Power Energy Syst., 106, pp. 105-123 (2019).

29. Anjos, M.F., Lodi, A., and Tanneau, M. "A decentralized framework for the optimal coordination of distributed energy resources" , IEEE Trans. Power Syst., 34(1), pp. 349-359 (2019).

30. Kraning, M., Chu, E., Lavaei, J., and Boyd, S. "Dynamic network energy management via proximal message passing" , Foundations and Trends@in Optimization, 1(2), pp. 73-126 (2014).

31. Kargarian, A., Fu, Y., DorMohammadi, S., et al. "Optimal operation of active distribution grids: a system of systems framework", IEEE Trans. Smart Grid, 5, pp. 1228-1237 (2014).

32. Zhao, B., Wang, X., Lin, D., et al. "Energy management of multiple microgrids based on a system of systems architecture", IEEE Trans. Power Syst., 33(6), pp. 6410-6421 (2018).

33. Xie, M., Ji, X., Hu, X., et al. "Autonomous optimized economic dispatch of active distribution system with multi-microgrids", Energy, 153, pp. 479-489 (2018). 
34. Kargarian, A., Mohammadi, J., Guo, J., et al. "Toward distributed/decentralized DC optimal power flow implementation in future electric power systems", IEEE Trans. Smart Grid, 9(4), pp. 2574-2594 (2018).

35. Zimmerman, R.D., Murillo-Sanchez, C.E., and Thomas, R.J. "MATPOWER's extensible optimal power flow architecture", in Proc. 2009 Power \& Energy Society General Meeting, Calgary, AB, Canada (2009).

36. Farivar, M. and Low, S.H. "Branch flow model: Relaxations and convexification-Part I", IEEE Trans. Power Syst., 28(3), pp. 2554-2564 (2013).

37. Kargarian, A., Fu, Y., and Li, Z. "Distributed securityconstrained unit commitment for large-scale power systems" , IEEE Trans. Power Syst., 30(4), pp. 19251936 (2015).

38. Kargarian, A., Mehrtash, M., and Falahati, B. "Decentralized implementation of unit commitment with analytical target cascading: A parallel approach", IEEE Trans. Power Syst, 33(4), pp. 3981-3993 (2018).

39. Baran, M.E. and Wu, F.F. "Network reconfiguration in distribution systems for loss reduction and load balancing", IEEE Trans. Power del., 4(2), pp. 14011407 (1989).

40. Lofberg, J. "Yalmip : A toolbox for modeling and optimization in Matlab", In Proceedings of the CACSD Conference, Taipei, Taiwan, pp. 284-289 (2004).

41. IBM ILOG CPLEX (2019). [Online]. Available: https://www.ibm.com/analytics/cplex-optimizer.

\section{Biographies}

Meysam Doostizadeh received the MSc and $\mathrm{PhD}$ degrees in Electrical Engineering from University of Tehran, Tehran, Iran, in 2012 and 2016, respectively. He is currently an Assistant Professor with the Faculty of Engineering, Lorestan University, Khorramabad, Iran. His research interests include electricity markets, smart grid technologies, and integration of renewable energy into power systems.

Mahmoud Reza Shakarami received his MSc and $\mathrm{PhD}$ degrees in Electrical Engineering from Iran University of Science and Technology, Tehran, Iran, in 2000 and 2010, respectively. He is currently an Associate Professor in Electrical Engineering Department of Lorestan University, Khorramabad, Iran. His current research interests are power system dynamics and stability, FACTS devices, and distribution systems.

Houman Bastami received his BS degree in Electrical Engineering from Lorestan University, Khorramabad, Iran, in 2011 and the MSc degree with honors in Electrical Engineering from Arak University, Arak, Iran, in 2015. He is currently a PhD candidate of Electrical Engineering at Lorestan University, Iran. His research interests include transactive energy market, smart grids, optimization techniques, and renewable energy modeling. 